\title{
Skeletonized inversion of surface wave: Active source versus controlled noise comparison
}

\author{
Jing $\mathrm{Li}^{1}$ and Sherif Hanafy ${ }^{1}$
}

\begin{abstract}
We have developed a skeletonized inversion method that inverts the S-wave velocity distribution from surface-wave dispersion curves. Instead of attempting to fit every wiggle in the surface waves with predicted data, it only inverts the picked dispersion curve, thereby mitigating the problem of getting stuck in a local minimum. We have applied this method to a synthetic model and seismic field data from Qademah fault, located at the western side of Saudi Arabia. For comparison, we have performed dispersion analysis for an active and controlled noise source seismic data that had some receivers in common with the passive array. The active and passive data show good agreement in the dispersive characteristics. Our results demonstrated that skeletonized inversion can obtain reliable 1D and 2D S-wave velocity models for our geologic setting. A limitation is that we need to build layered initial model to calculate the Jacobian matrix, which is time consuming.
\end{abstract}

\section{Introduction}

The surface-wave method has been progressively established as an effective and reliable tool for improving the seismic imaging with different scales (Roy et al., 2010; Socco et al., 2010). The energy of the surface wave is dissipated proportionally to the distance from source (Nazarizan et al., 1983), and its propagation paths are concentrated on the depths according to its wavelength (Haney and Tsai, 2015). In a near-surface application, the surface wave is dispersive in heterogeneous media (Thomson, 1950) and only highly sensitive to the S-wave velocity and the depth; it can be used to estimate the Swave velocity structure. It is also time and cost effective, especially for passive data, which can only retrieve surface waves (Hanafy et al., 2015). In addition, in some cases, seismic velocities decrease with depth, such as permafrost structures, underground cavities, etc. In this case, refraction waves are often unable to provide accurate information (Trupp et al., 2009). In contrast, the surface-wave method can be used to investigate sites with low-velocity layers, which is difficult to do with the refraction method.

Surface-wave inversion methods fall into two categories: (1) the classic method of inverting dispersion curves for a 1D layered medium (Evison et al., 1959; Nazarizan et al., 1983; Park et al., 1998; Xia et al., 2004) and (2) fullwaveform inversion (FWI) (Groos et al., 2014; Solano et al., 2014) for 2D and 3D media. The classic method robustly inverts for a 1D S-wave velocity model, but it becomes less accurate with increasing lateral heterogeneity in the subsurface. In comparison, FWI can theo- retically account for any lateral heterogeneity, but it is computationally expensive and can easily get stuck in local minima associated with the objective function.

To mitigate the classic method's restriction to a layered medium and also to avoid FWI's sensitivity to local minima, we present a skeletonized inversion method that inverts the dispersion curves for $2 \mathrm{D}$ or $3 \mathrm{D}$ velocity models. The picked dispersion curves are skeletonized versions of the data that tend to make the objective function simpler, and hence this new method has better convergence properties than does FWI. The idea of skeletonizing the data is borrowed from Luo and Schuster (1991a, 1991b), who use the wave equation to invert $\mathrm{P}$-wave traveltime, which are skeletonized representations of the data. They find that wave-equation traveltime inversion (WT) enjoyed robust convergence properties and also overcame the high-frequency limitation of raytracing tomography. Similarly, we now present waveequation dispersion inversion (WD) to mitigate some of the convergence problems associated with FWI of surface waves.

We first present the workflow of skeletonized inversion and discuss its benefits and limitations. The method is then tested on synthetic and field data recorded near the Qademah fault at the western side of Saudi Arabia. The final section presents a summary of our work and conclusion.

\section{Method of skeletonized inversion}

The classic 1D surface-wave inversion can only be applied in stratified medium. Unfortunately, in some

\footnotetext{
${ }^{1}$ King Abdullah University of Science and Technology (KAUST), Thuwal, Saudi Arabia. E-mail: jing.li@kaust.edu.sa; sherif.mahmoud@kaust.edu.sa. Manuscript received by the Editor 30 October 2015; revised manuscript received 24 March 2016; published online 12 July 2016 . This paper appears in Interpretation, Vol. 4, No. 3 (August 2016); p. SH11-SH19, 10 FIGS.

http://dx.doi.org/10.1190/INT-2015-0174.1. @ 2016 Society of Exploration Geophysicists and American Association of Petroleum Geologists. All rights reserved.
} 
areas, the lateral heterogeneity is too strong to assume a layered medium. This problem is addressed by skeletonized inversion of surface waves (Schuster, 2015; Zhang et al., 2015; J. Li and G. T. Schuster, personal communication, 2016) because the elastic-wave equation is used to invert the dispersion curves for $2 \mathrm{D}$ and $3 \mathrm{D}$ velocity models. It uses the wave equation and an iterative gradient optimization algorithm to invert the dispersion curve. To do that, we first pick the fundamental dispersion velocity $C(\omega)_{\text {obs }}$ from the recorded data. Then, we use a linear Radon transform (LRT) method to improve the overall resolution of dispersive images. These two-steps process improves the dispersion image by more than $50 \%$ relative to the slant stacking algorithms (Luo et al., 2008) and can transform the signal energy to $\tau-p$ domain and filter the noise signal effectively (see Appendix A).

A weighted least-squared inversion strategy is used to invert the dispersion curve for the S-wave velocity distribution. Here, we have a nonlinear function; however, it can be linearized by Taylor expansion to use the matrix theory (Xia et al., 1999):

$$
J \Delta x=\Delta b,
$$

where $\Delta b$ is the difference between the observed and predicted phase velocity estimated from the recorded data, $\Delta x$ is the residual of S-wave velocity at each iteration, and $J$ is the Jacobian matrix. The iterative leastsquared inversion uses regularization (Marquardt, 1963) and the singular value decomposition (SVD) technique (Golub and Reinsch, 1970). The objective function is defined as

$$
\phi=\|L(J \Delta x-\Delta b)\|_{2}^{2}+\lambda\|\Delta x\|_{2}^{2},
$$

where \|\|$_{2}$ is the $l_{2}$-norm length of a vector, $\lambda$ is the damping factor, $L$ is related to the weighting matrix $W$ determined by errors in phase velocities, so the iterative $\mathrm{S}$-wave velocity solution is given by

$$
v_{s}\left(x^{k+1}\right)=v_{s}\left(x^{k}\right)-\alpha \Delta x,
$$

and $\Delta x$ is the solution of equation 2. Defining the matrix $A=L J$ and applying the SVD technique $A=U \Lambda V^{T}$

$$
\Delta x=V\left(\Lambda^{2}+\lambda I\right)^{-1} \Lambda U^{T} d .
$$

The classic 1D inversion can be established for a stratified medium with homogeneous linear elastic layers, using a matrix formulation for a single layer and then building the global matrix that governs the problem (Socco et al., 2010). Here, the Jacobian matrix is equal to the partial derivative of dispersion curve, which can be calculated by the finite-difference wave equation

$$
J=\frac{\partial C(\omega)}{\partial c_{i}} \approx \frac{C(\omega)_{c+\delta c}-C(\omega)_{c}}{\delta_{C_{i}}},
$$

where $c$ is the reference S-wave velocity model and $\delta c_{i}$ is the perturbed velocity of the $i$ th layer. Two finite-differ- ence simulations are required to compute $\partial C(\omega) / \partial_{C_{i}}$ : one for the predicted S-wave velocity model and one for the S-wave velocity model in the $i$ th layer perturbed by $\delta c_{i}$.

\section{D layer synthetic model test}

Two synthetic simulations are used to test the effectiveness of the skeletonized inversion method. One test is for a two-layer model for LRT to extract the dispersion curve, and the other is a comparison test for a conventional 1D inversion and our skeletonized inversion model.

\section{Linear Radon transform method test}

Generating a reliable dispersion curve is one of the critical steps in surface-wave inversion. We need not only to pick the dispersion curve but also to separate the fundamental wave from the high-order energy (Luo et al., 2007). For data with a low signal-to-noise ratio, the traditional methods, such as the $f-k$ transformation (Yilmaz, 1987), the phase shift (Park et al., 1998), or the slant stacking algorithm (Xia et al., 2007) cannot obtain satisfactory results due to resolution loss, aliasing, and a limited recording aperture (Trad et al., 2003).

The following simple two-layer model is used to test the accuracy of extracting dispersion curves from surface-wave data. The first-layer velocities are $V_{\mathrm{P}}=$ $800 \mathrm{~m} / \mathrm{s}, V_{\mathrm{S}}=200 \mathrm{~m} / \mathrm{s}$, and the half-space velocities are $V_{\mathrm{P}}=1200 \mathrm{~m} / \mathrm{s}$ and $V_{\mathrm{S}}=400 \mathrm{~m} / \mathrm{s}$; the density is $2600 \mathrm{~kg} / \mathrm{m}^{3}$. The thickness of the surface layer is $10 \mathrm{~m}$. We use $2 \mathrm{D}$ elastic wave finite-difference time domain (FDTD) code (Li et al., 2012) with a free-surface condition to simulate the surface-wave response with a Ricker wavelet centered at $20 \mathrm{~Hz}$. The two-layer model size is $30 \times 60 \mathrm{~m}$ in vertical and horizontal direction, respectively, and the grid size is $0.5 \mathrm{~m}$. We generated 60 shot gathers; each one has 60 traces with a shot and trace interval equal to $1 \mathrm{~m}$. The total recording time is $0.5 \mathrm{~s}$ at a $1 \mathrm{~ms}$ sample interval. Figure $1 \mathrm{a}$ is a sample shot gather that shows clear surface-wave dispersion, and Figure 1b shows the dispersion curve of the synthetic shot gather. The computed dispersion curve with the LRT result is almost in agreement with that of the analytical dispersion curve (Hisada, 1994).

\section{$1 D$ conventional inversion versus skeletonized inversion}

We now compare the skeletonized inversion method with traditional 1D dispersion curve inversion (Park et al., 1998) for a $1 \mathrm{D}$ synthetic model. The synthetic model consists of three layers with the parameters shown in Figure 2a. A shot gather (Figure 2b) is simulated by an FDTD solution to the 2D elastic-wave equation. Here, the traces are recorded for the vertical component on the free surface. The source wavelet is a Ricker wavelet peaked at $20 \mathrm{~Hz}$. From the synthetic data (Figure 2b), we extracted the dispersion curve using the LRT method (Figure 2c). To test the inversion accuracy, we start the inversion with a smooth initial 
velocity model (Figure 2f). As shown in Figure 2d, the misfit is reduced to 0.3 after eight iterations and the observed data mostly agree with the predicted data for both methods (Figure 2e). Figure 2f shows the S-wave velocity for the $1 \mathrm{D}$ and $2 \mathrm{D}$ inversions. Here, the $2 \mathrm{D}$ result is almost in agreement with the true model, whereas the $1 \mathrm{D}$ result has some error.

\section{Active and controlled noise seismic data inversion}

We recorded a controlled noise source (CNS) data set, where we used a noise-making truck (shown in Figure 3a) as a seismic source. The recorded seismic data set has 60 vertical-component receivers with receiver interval of $10 \mathrm{~m}$. To record the CNS data, we drove a truck (Figure 3a) around the receiver's line for 2 hours, and the seismic noise from the truck is recorded at each receiver location. The raw data are broken up into eight 15 min windows, then we select a master trace, the $15 \mathrm{~min}$ window of the master trace is correlated with the corresponding windows of all other traces. The resulting correlograms from each window are stacked onto those from the other windows to generate a virtual shot gather with virtual shot located at the master trace. The whole process is repeated with other traces as the master trace to create the other virtual shot gathers. The same receiver line is also used to record active-source seismic data with shot interval of $5 \mathrm{~m}$. To generate the active-source seismic energy, we used an accelerated weight drop (40 kgm; Figure 3b). Figure 4c shows a sample of the recorded active-source shot gathers. The CNS raw data (Figure 4a) and active-source raw data (Figure 4c) show obvious surface wave. With the LRT processing, shot gather is transformed from the $t-x$ domain to the $\tau$-velocity domain, and the surface wave can be separate according to the velocity difference between the $\mathrm{P}$ - and the surface-wave velocity values. Figure 5 is the frequency spectrum of the data shown in Figure 4. The blue and red lines are the raw and processed data, respectively, with LRT. The surface-wave frequency bandwidth ranges between 5 and $20 \mathrm{~Hz}$. Comparing dispersion curves of CNS data (Figure 6) with that of the active source data (Figure 7) shows that the dispersion curve of surface waves al- most match each other; however, some virtual shot gathers do not have the same amplitude levels as the active shot gathers. This problem is partly mitigated if the

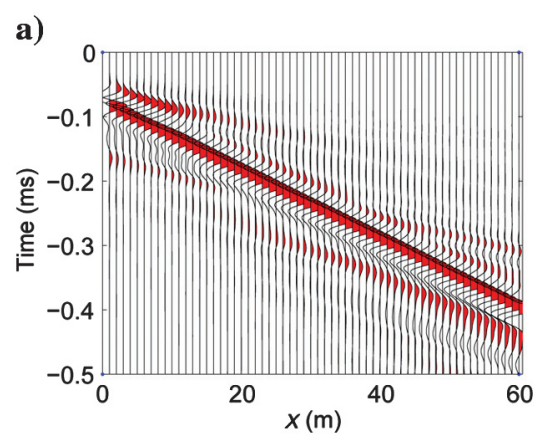

b)

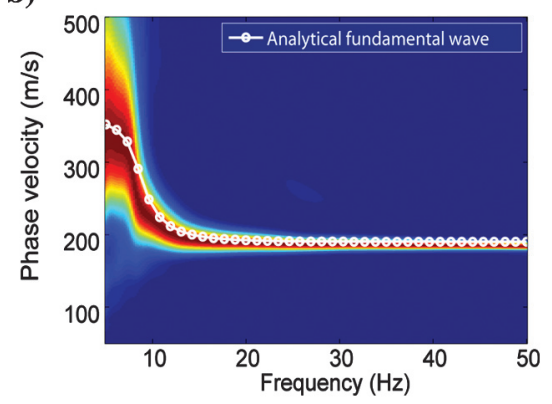

Figure 1. (a) Synthetic shot gather; (b) LRT and analytic (dashed white lines) dispersion curves.

a)

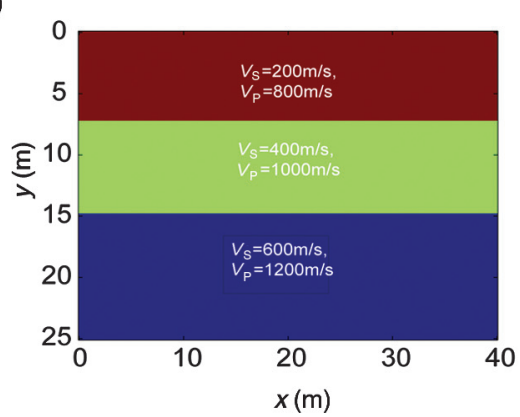

c)

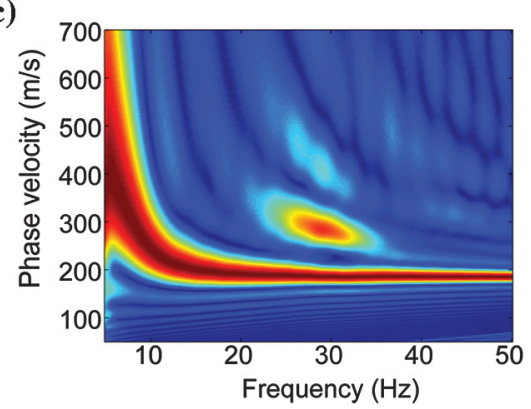

e)

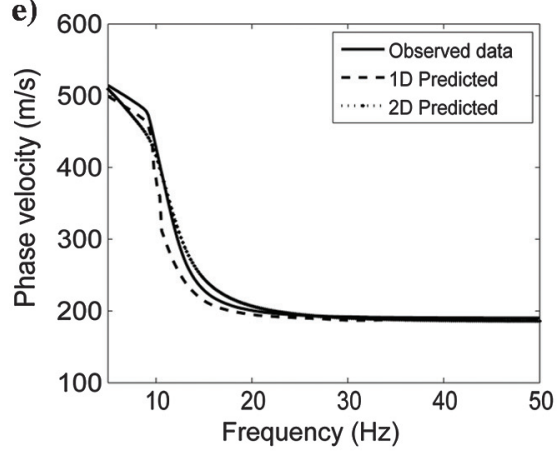

b)

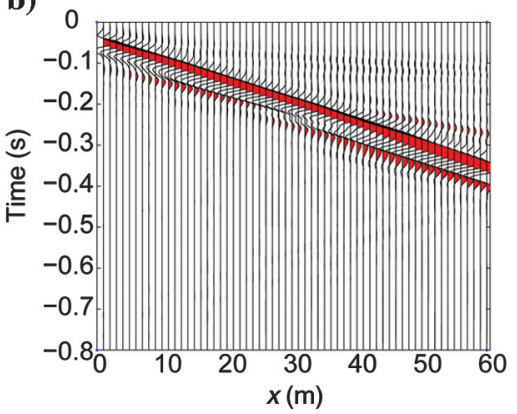

d)

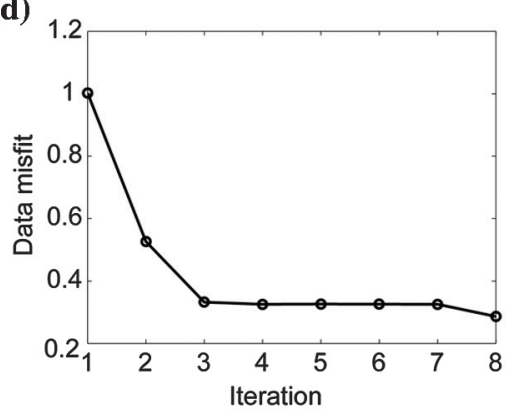

f)

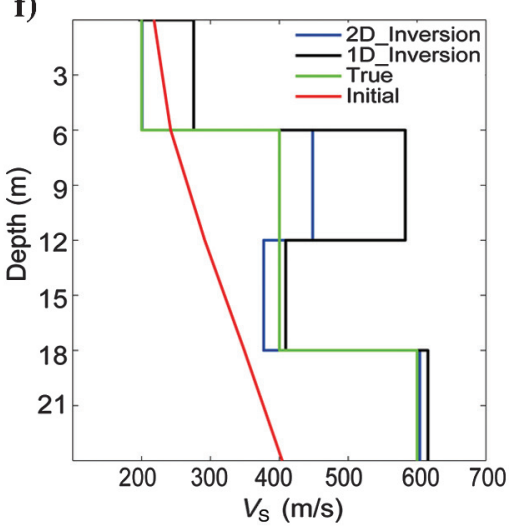

Figure 2. Comparison of skeletonized inversion method and traditional 1D inversion with synthetic data. (a) Synthetic model; (b) shot gather; (c) dispersion curve with LRT; (d) data misfit; (e) phase velocities of the observed and predicted data; and (f) S-wave velocity inversion results. 
acausal part of the virtual trace is mirrored across the time-axis and added to the causal part (Van Dalen et al., 2013; Forghani-Arani et al., 2014). The six dispersion curves in Figures 6 and 7 indicate the average phasewave velocity at shots number $1,5,15,20,25$, and 35 located at offsets 100, 150, 250, 300, 350, and $450 \mathrm{~m}$, respectively. From the dispersion curve, we can conclude some velocity information of the substructure as follows:

1) All dispersion curves show the fundamental wave. However, only dispersion curves in Figures 6a-6f and $7 \mathrm{a}-7 \mathrm{c}$ show the first-order wave, which indicates that a low-velocity layer and/or a near-surface high-velocity contrast exist.

2) The dispersion curves also demonstrate that the 1st, 5th, and 15th shots (a, b, and c) have a cutfrequency for first high-order dispersion curve in the $5-20 \mathrm{~Hz}$ range. Referring to the theoretical formula of the cut frequency (Xia et al., 2007)

$$
f_{n}=\frac{n v_{s 1}}{2 h \sqrt{1-\left(\frac{v_{s 1}}{v_{s 2}}\right)^{2}}},
$$

where $V_{\mathrm{S} 1}$ and $V_{\mathrm{S} 2}$ are the S-wave velocities of the top and the halfspace (consider a two-layer model), respectively; $h$ is the thickness of the top layer; and $f_{n}$ is the cut-off frequency of the $n$th high mode. It indicates that either the thickness of the shallow layer is greater or the shallow S-wave velocity is smaller at the 1st, 5th, and 15th shot locations relative to the other locations.

3) In some cases, such as Figures $6 a-6 c$ and $7 \mathrm{a}-7 \mathrm{c}$, we notice that the fundamental mode disappears in a certain frequency range $(7-13 \mathrm{~Hz}$ in this case), and the first-order mode is shown with high amplitude. This cannot be explained with the low- velocity layer model as shown on the synthetic model discussed in Figure 2. A second synthetic model is used to explain this observation; the synthetic model has three layers with lateral velocity change in the second layer (Figure 8a). The first layer has an S-wave velocity of $300 \mathrm{~m} / \mathrm{s}$, but the second layer has a sharp velocity change in the horizontal direction from 200 to $450 \mathrm{~m} / \mathrm{s}$. The half-space $\mathrm{S}$-wave velocity is $600 \mathrm{~m} / \mathrm{s}$. The source center frequency is $30 \mathrm{~Hz}$ Ricker wavelet. The surface-wave dispersion curve (Figure 8b) shows similar characteristics as that shown in Figures $6 a-6 c$ and $7 a-7 c$ from the field data set. The result of the second synthetic model suggests that the field site has a lowvelocity layer with a sharp lateral velocity changes, which are shown on the dispersion curve as the
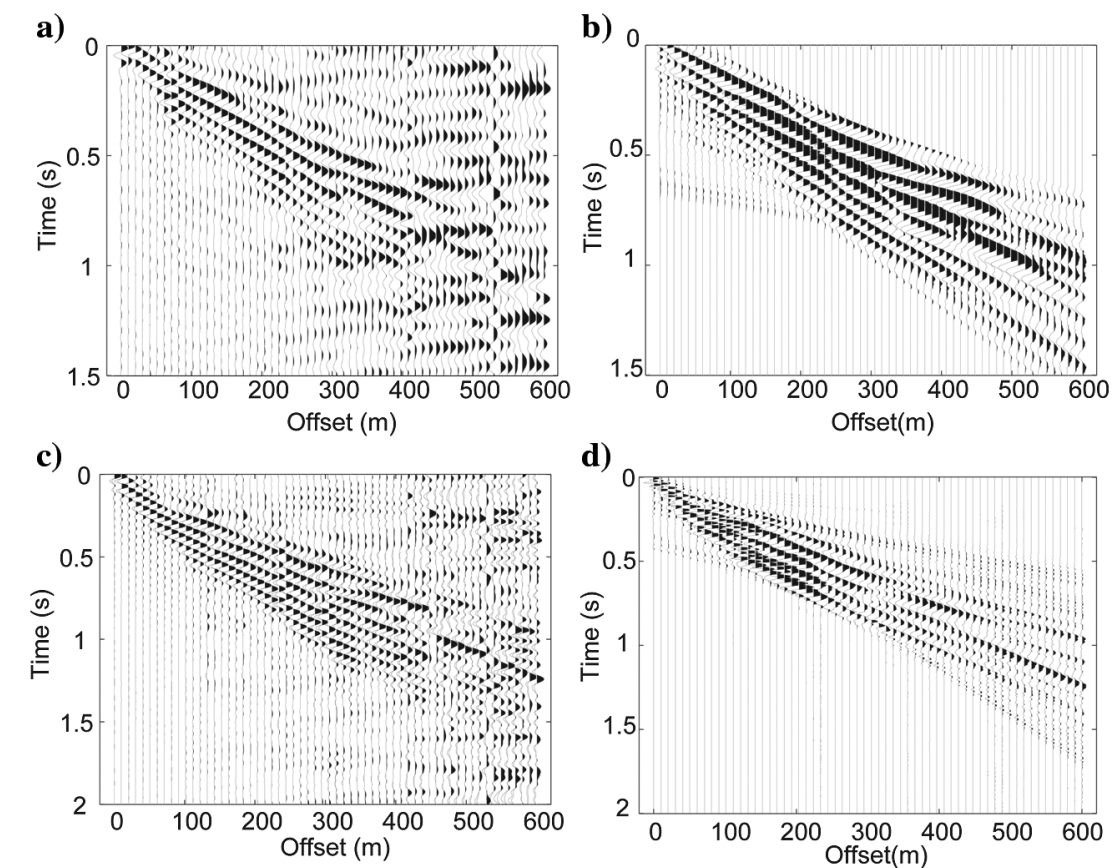

d)

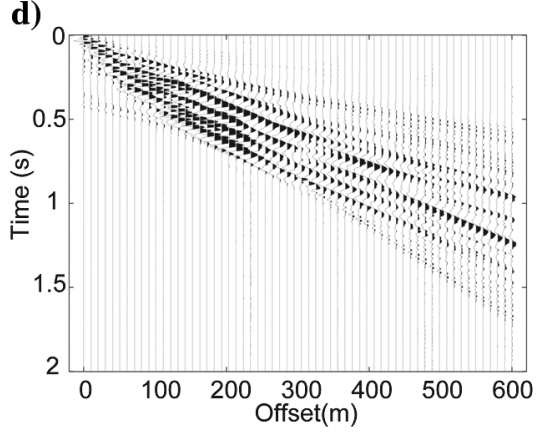

Figure 4. The surface wave separated from controlled noise and active seismic data with Radon transform (RT). (a) CNS raw data; (b) surface wave with RT; (c) active-source raw data; and (d) surface wave with RT.

a)

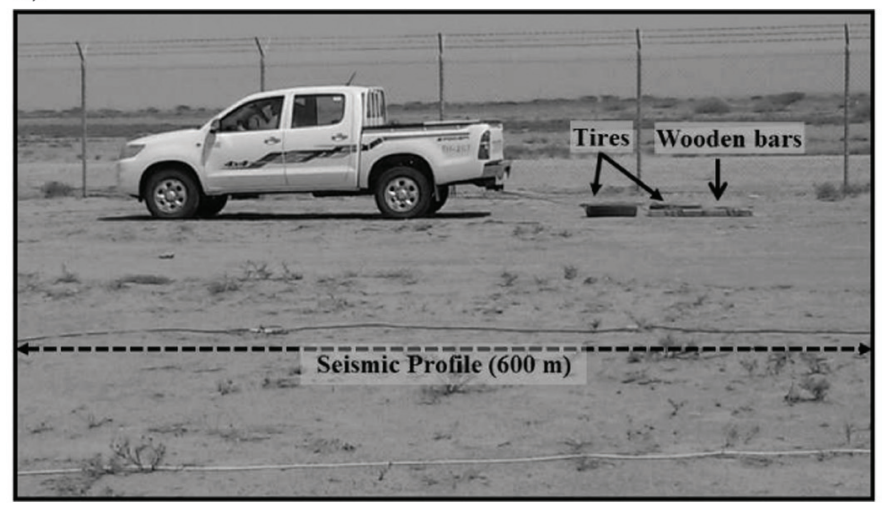

b)

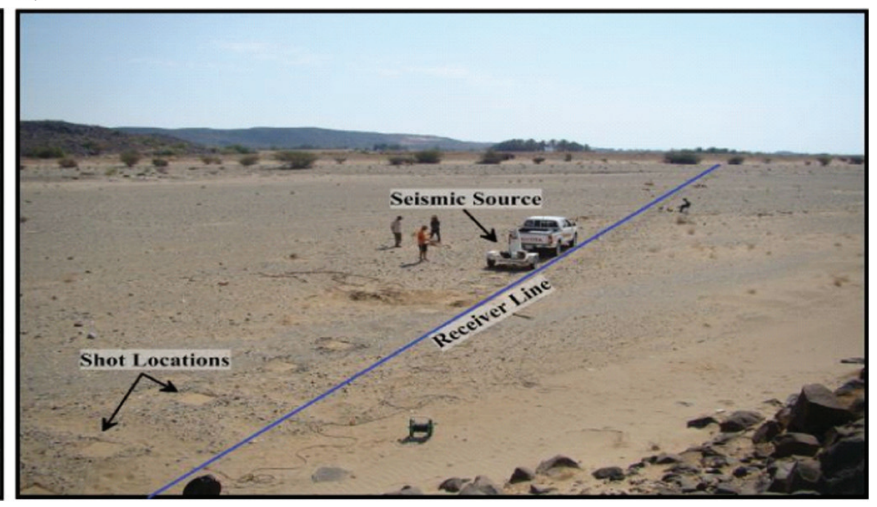

Figure 3. A photo taken during data survey for (a) control noise and (b) active-source seismic. 
absence of fundamental mode and high amplitude of the first-order mode.

According to the single shot dispersion curve result (Figures 6 and 7), we used the LRT to extract the dispersion curves of all data and then inverted it to generate the S-wave velocity by the WD method. The LRT method uses 20 traces starting from the shot position to estimate the dispersion curve of each shot gather. The transformed data are then displayed in phase-velocity space, and the dispersion curve is picked for the fundamental wave. The dispersion data are inverted by the WD method, and the resulting S-wave velocity profile

a)

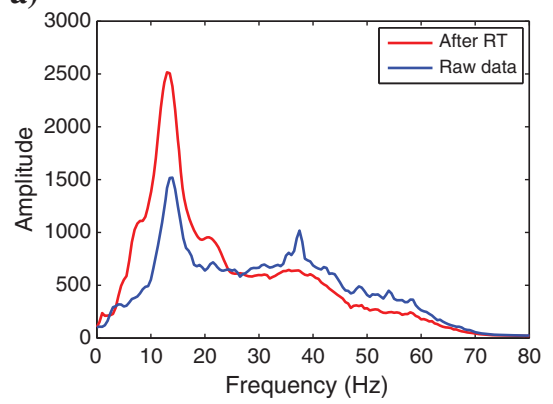

b)

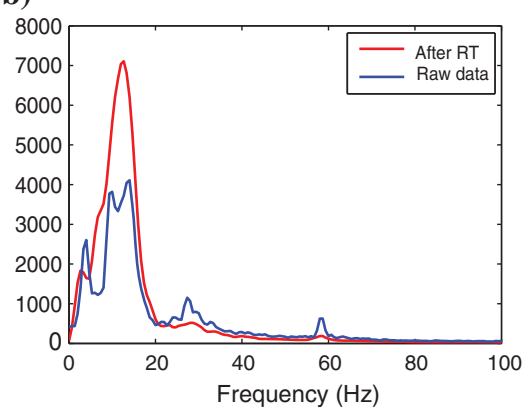

Figure 5. Comparison of frequency spectrum of (a) active source and (b) CNS. Here, the blue line represents the raw data and the red line represents the RT result.

a)

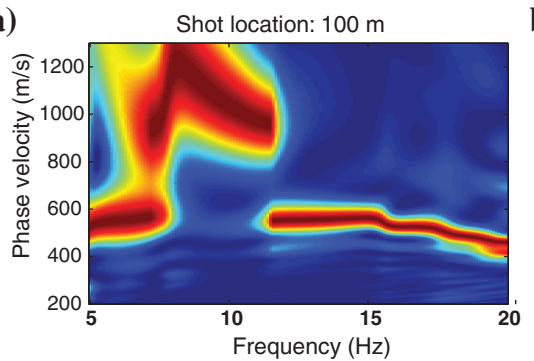

c)

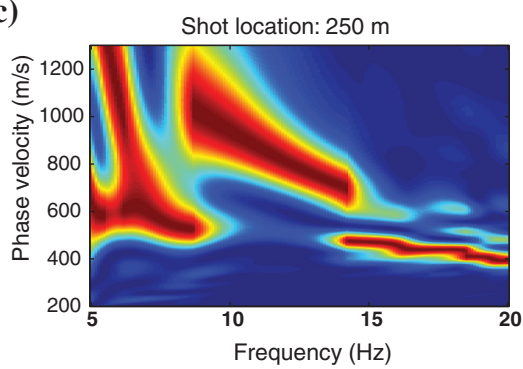

e)

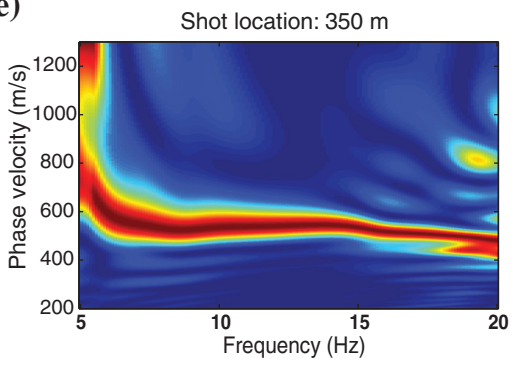

b)

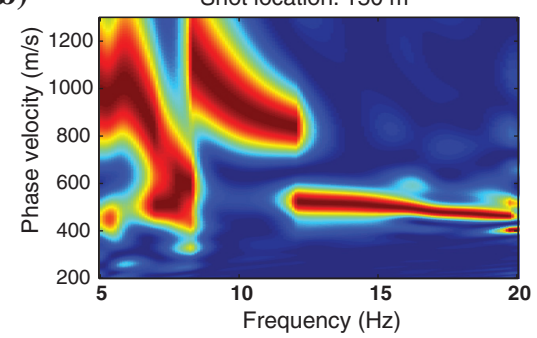

d)

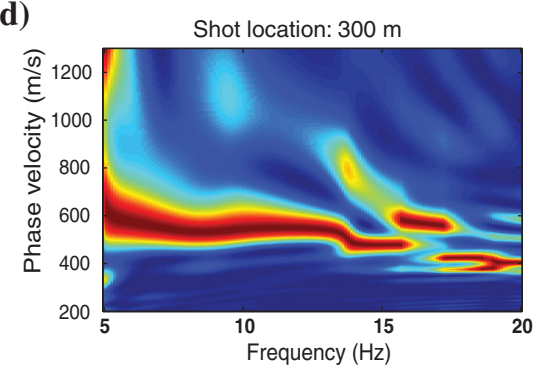

f)

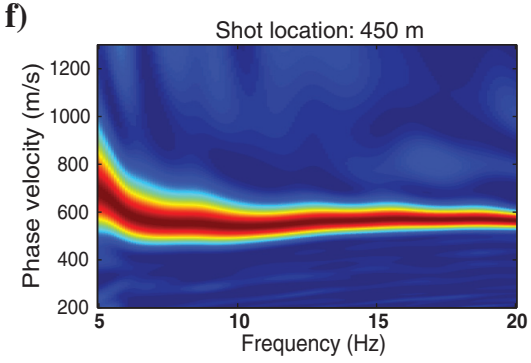

is centered at the 20 traces. This procedure is rolled from one master shot position to the next until all shot positions in the offset range $100<x<500 \mathrm{~m}$ are sampled.

Figure 9a shows the common offset gather (COG) of the recorded active-source data set, Figure $9 \mathrm{~b}$ shows the 2D CNS phase velocity image, and Figure 9c shows the $2 \mathrm{D}$ active-source phase velocity image. The anomaly shown on the COG profile (offset range, $100-300 \mathrm{~m}$ and time $0.5 \mathrm{~s}$ ) is also shown in the phase wave velocity images (offset range, 100-300 m and frequency $12 \mathrm{~Hz}$ ), which indicates that the active-source (Figure $9 \mathrm{~b}$ ) and CNS (Figure 9c) results are consistent with the COG profile (Figure 9a). In addition, the active and CNS phase velocity structures are similar to one another except at offset range of 100-300 $\mathrm{m}$ and frequency range of $10-5 \mathrm{~Hz}$. This could be due to the different number of shots in each data set (120 CSGs in the active source data set and 60 CSGs in the CNS data set). The $2 \mathrm{D}$ phase-velocity images are inverted to generate the corresponding S-wave tomograms using $2 \mathrm{D}$ skeletonized surface-wave inversion. The frequency range of the surface wave is $5-20 \mathrm{~Hz}$. According to the relationship between the phase velocity $f$ and the penetration depth $z\left(z=v_{R} / 3 f\right.$, where $v_{R}$ is the phase

Figure 6. The dispersion curves computed from controlled noise seismic data at six different locations: (a) shots 1, (b) 5, (c) 15, (d) 20, (e) 25, and (f) 35 . 
Figure 7. The dispersion curves computed from active source seismic data at six different locations: (a) shots 1, (b) 5, (c) 15, (d) 20, (e) 25, and (f) 35 .

Figure 8. The second synthetic test: (a) velocity model and (b) the dispersion curve.

Figure 9. (a) The COG and the phase velocity tomograms for the (b) active and (c) controlled noise surveys. a)

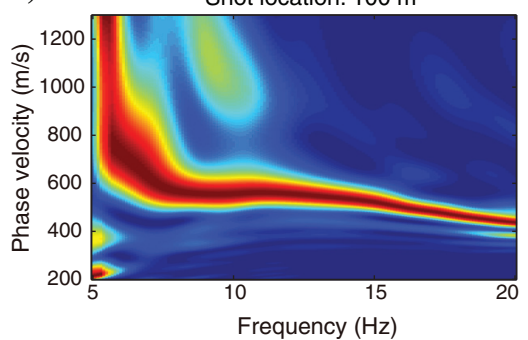

c)

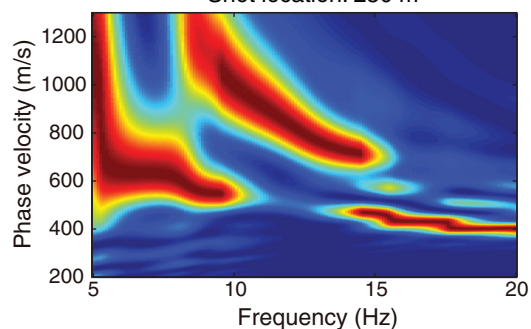

e)

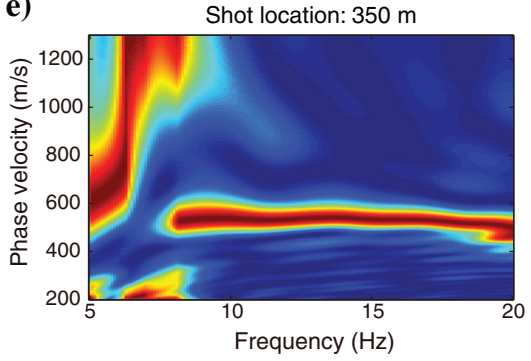

a)

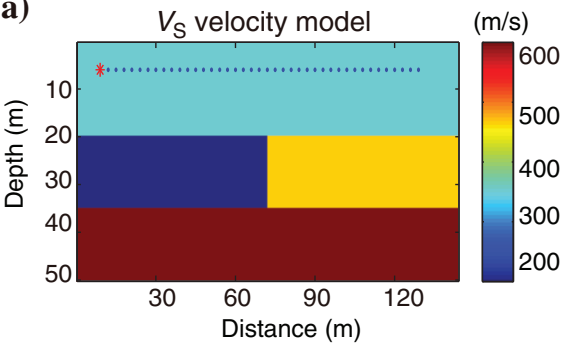

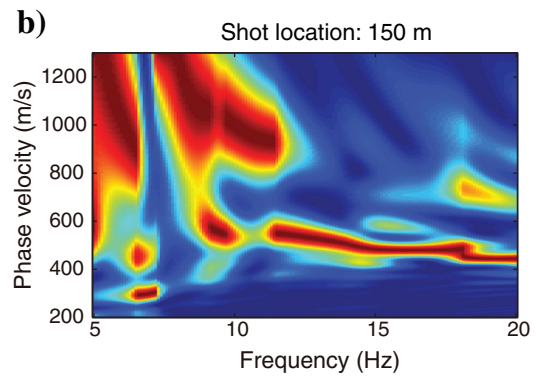

d)

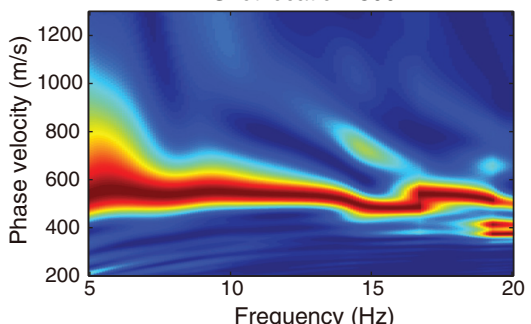

f)

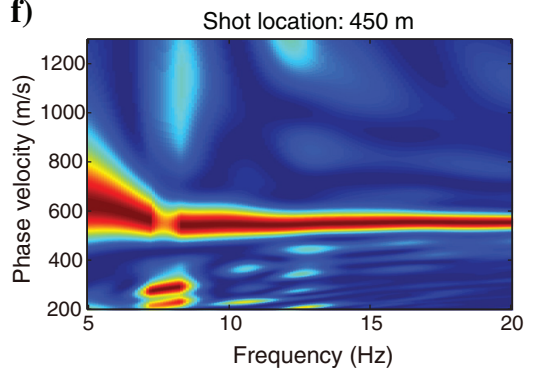

b)

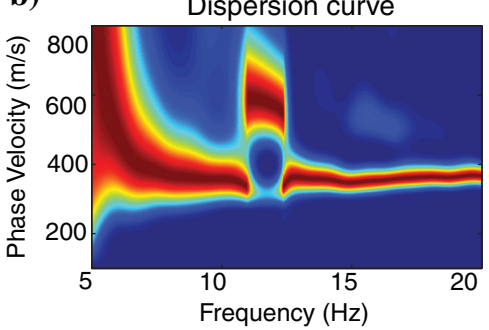

a)

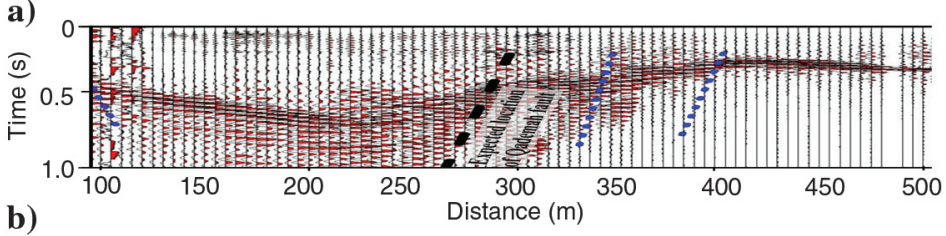

b)

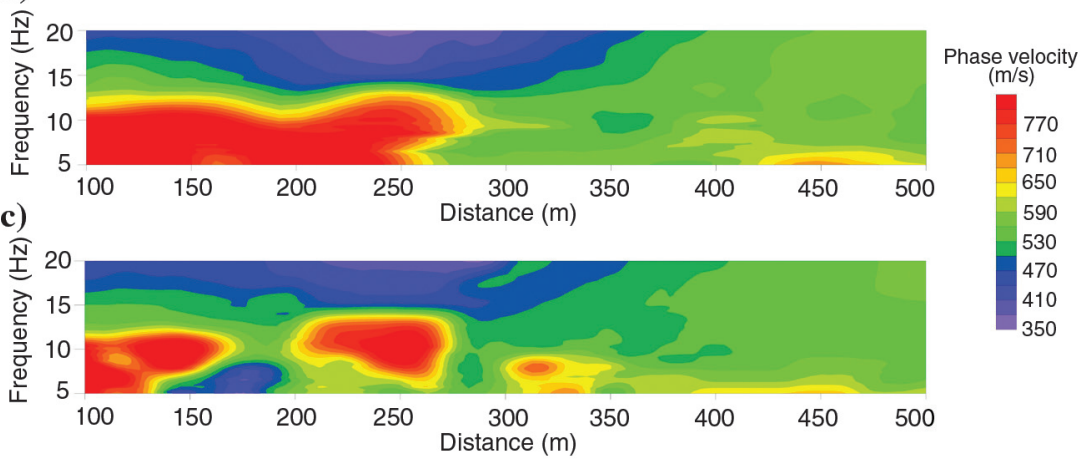



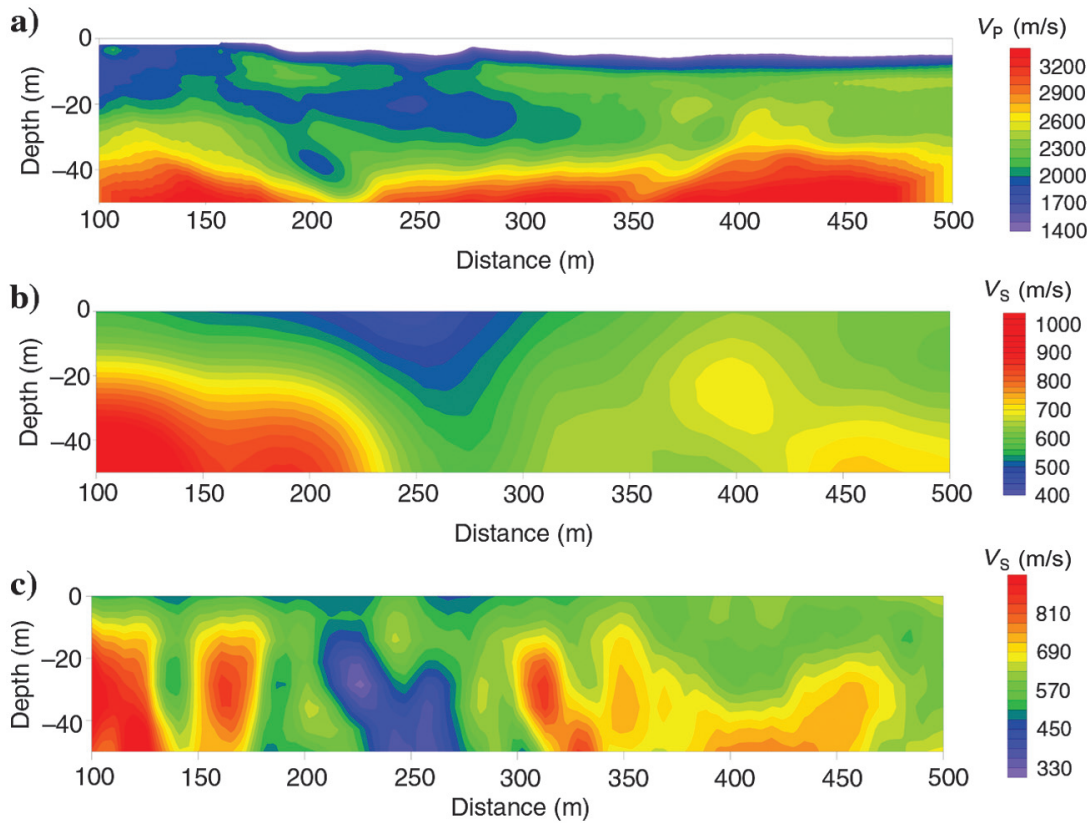

Figure 10. Comparison between the (a) P-wave velocity, (b) S-wave velocity tomograms from CNS data, and (c) S-wave velocity tomogram from activesource data.

velocity), the maximum penetration depth is approximately $50 \mathrm{~m}$. The inverted $2 \mathrm{D}$ S-wave velocity tomograms and the P-wave velocity tomogram are shown in Figure 10 . Note that the P-wave velocity tomogram is almost in agreement with the inverted S-wave velocity tomograms from CNS and active source data sets. A low-velocity anomaly is shown between offsets 220 and $300 \mathrm{~m}$ (Figure 10b and 10c). According to the P-wave tomogram and geology survey, this low-velocity anomaly is corresponding to the colluvial wedge associated with the Qademah fault. The depth to the low-velocity anomaly in the $\mathrm{P}$-wave velocity tomogram is approximately consistent with that of the S-wave tomograms. A high-velocity layer ( $>3000 \mathrm{~m} / \mathrm{s}$ at depth $>40 \mathrm{~m}$ ) is shown on the P-wave tomogram (Figure 10a), which is not shown on the S-wave tomogram, this could be due to the shallow penetration depth of the surface waves.

\section{Conclusions}

The skeletonized inversion method overcomes some of the limitations of the conventional 1D S-wave inversion method and mitigates the FWI convergence problems of the surface waves. It is efficient for $1 \mathrm{D}$ and $2 \mathrm{D}$ models. The LRT method is used to extract the dispersion curve from surface-wave data over a wide frequency range. Our $1 \mathrm{D}$ and $2 \mathrm{D}$ tests suggest that the skeletonized inversion method is reliable and has a higher accuracy compared with traditional 1D inversion. In addition, it is efficient for a limited number of layers, and is applicable to $2 \mathrm{D}$ and $3 \mathrm{D}$ velocity models. The activesource and CNS seismic data tests suggest that the result of the skeletonized S-wave velocity inversion is in agreement with the $\mathrm{P}$-wave tomogram and has high resolution for shallow subsurface S-wave velocity structures. The proposed method not only has a good vertical resolution for models with velocity increases with depth, but also suits the velocity reversal structure.

The skeletonized inversion technique uses a layered media to build the initial velocity model. To calculate the Jacobian matrix, for an $N-1$ layer velocity model, we need to do $N+1$ finite-difference simulations for one shot, which is time-consuming. One of the implicit assumptions is that the S-wave velocity model is simple enough to produce a fundamental Rayleigh curve that is easily pickable. This assumption will be violated in more complex models and can make it difficult to implement the skeletonized inversion method. In the future, work on the skeletonized surface-wave inversion with the adjoint state method is developed that avoids the generation of an undetermined system of equations.

\section{Acknowledgments}

We thank the sponsors for supporting the Center for Subsurface Imaging and Fluid Modeling (CSIM). We thank KAUST for funding this research.

\section{Appendix A}

\section{Basic theory of Radon transform}

The forward step of LRT is written as

$$
d(x, t)=\sum_{p_{\min }}^{p_{\max }} m(p, \tau=t-p x),
$$

where $d(x, t)$ is the trace recorded at $x$ and $m(p, \tau)$ is the offset and adjoint transformation,

respectively. Here, $p$ is the slowness parameter. In the frequency domain, the LRT can be calculated for each frequency $f$ :

$$
d(x, t)=\sum_{p_{\min }}^{p_{\max }} m(p, f) e^{i 2 \pi f p x} .
$$

and

$$
m(p, f)=\sum_{x_{\min }}^{x_{\max }} d(x, t) e^{-i 2 \pi f p x} .
$$

According to previous studies (Luo et al., 2008), the LRT workflow includes three steps:

1) Transform the shot gather from the $t-x$ domain into the $f-x$ domain. 
2) The LRT is completed for each frequency, and the data are transformed from the $f-x$ domain to the $f-p$ domain.

3) Change into the $f-v$ domain with a linear interpolation operation.

The amplitudes will largely be preserved if the computation is done properly within the bandwidth of the signal (Nowak and Imhof, 2006).

\section{References}

Evison, F. F., R. Orr, and C. Ingham, 1959, Thickness of the earth's crust in Antarctica: Nature, 183, 306-308, doi: 10 .1038/183306a0.

Forghani-Arani, F., M. Willis, R. Snieder, S. S. Haines, J. Behura, M. Batzle, and M. Davidson, 2014, Dispersion analysis of passive surface-wave noise generated during hydraulic-fracturing operations: Journal of Applied Geophysics, 111, 129-134, doi: 10.1016/j.jappgeo.2014 .09.008.

Golub, G. H., and C. Reinsch, 1970, Singular value decomposition and least squares solutions: Numerische Mathematik, 14, 403-420, doi: 10.1007/BF02163027.

Groos, L., M. Schfer, T. Forbriger, and T. Bohlen, 2014, The role of attenuation in 2D full-waveform inversion of shallow-seismic body and Rayleigh waves: Geophysics, 79, no. 6, R247-R261, doi: 10.1190/geo2013-0462.1.

Hanafy, S. M., A. AlTheyab, and G. T. Schuster, 2015, Controlled noise seismology: 85th Annual International Meeting, SEG, Expanded Abstracts, 5102-5206.

Haney, M. M., and V. C. Tsai, 2015, Nonperturbational surface-wave inversion: A Dix-type relation for surface waves: Geophysics, 80, no. 6, EN167-EN177, doi: 10 .1190/geo2014-0612.1.

Hisada, Y., 1994, An efficient method for computing green's functions for a layered half-space with sources and receivers at close depths: Bulletin of the Seismological Society of America, 84, 1456-1472.

Li, J., Z. F. Zeng, L. Huang, and F. S. Liu, 2012, GPR simulation based on complex frequency shifted recursive integration PML boundary of 3D high order FDTD: Computers and Geoscience, 49, 121-130, doi: 10.1016/j. cageo.2012.06.020.

Luo, Y., and G. T. Schuster, 1991a, Wave equation inversion of skeletalized geophysical data: Geophysical Journal International, 105, 289-294, doi: 10.1111/gji.1991.105. issue-2.

Luo, Y., and G. T. Schuster, 1991b, Wave-equation traveltime inversion: Geophysics, 56, 645-653, doi: 10.1190/1.1443081.

Luo, Y., J. Xia, J. Liu, Q. Liu, and S. Xu, 2007, Joint inversion of high-frequency surface waves with fundamental and higher modes: Journal of Applied Geophysics, 62, 375384, doi: 10.1016/j.jappgeo.2007.02.004.

Luo, Y., J. Xia, R. D. Miller, Y. Xu, J. Liu, and Q. Liu, 2008, Rayleigh-wave dispersive energy imaging using a highresolution linear radon transform: Pure and Applied Geophysics, 165, 903-922, doi: 10.1007/s00024-008-0338-4.
Marquardt, D. W., 1963, An algorithm for least-squares estimation of nonlinear parameters: Journal of the Society for Industrial and Applied Mathematics, 11, 431-441, doi: $10.1137 / 0111030$.

Nazarizan, S., K. H. Stokoe, II, and W. Hudson, 1983, Use of spectral analysis of surface waves method for determination of moduli and thickness of pavement systems: Transportation Research Record, 930, 38-45.

Nowak, E. J., and M. G. Imhof, 2006, Amplitude preservation of radon-based multiple-removal filters: Geophysics, 71, no. 5, V123-V126, doi: 10.1190/1.2243711.

Park, C. B., R. D. Miller, and J. Xia, 1998, Imaging dispersion curves of surface waves on multi-channel record: 68th Annual International Meeting, SEG, Expanded Abstracts, 1377-1380.

Roy, S., R. R. Stewart, and K. Al Dulaijan, 2010, S-wave velocity and statics from groundroll inversion: The Leading Edge, 29, 1250-1257, doi: 10.1190/1.3496915.

Schonewille, M., and A. Duijndam, 2001, Parabolic radon transform, sampling and efficiency: Geophysics, 66, 667-678, doi: 10.1190/1.1444957.

Schuster, G., 2015, Advance seismic inversion: SEG.

Socco, L. V., S. Foti, and D. Boiero, 2010, Surface-wave analysis for building near-surface velocity models: Established approaches and new perspectives: Geophysics, 75, no. 5, 75A83-75A102, doi: 10.1190/1.3479491.

Solano, C. P., D. Donno, and H. Chauris, 2014, Alternative waveform inversion for surface wave analysis in 2-D media: Geophysical Journal International, 198, 13591372, doi: 10.1093/gji/ggu211.

Thomson, W. T., 1950, Transmission of elastic waves through a stratified solid medium: Journal of Applied Physics, 21, 89-93, doi: 10.1063/1.1699629.

Trad, D., T. Ulrych, and M. Sacchi, 2003, Latest views of the sparse radon transform: Geophysics, 68, 386-399, doi: 10.1190/1.1543224.

Trupp, R., J. Hastings, S. Cheadle, and R. Vesely, 2009, Seismic in arctic environs: Meeting the challenge: The Leading Edge, 28, 936-942.

Van Dalen, K. N., K. Wapenaar, and D. F. Halliday, 2013, Surface wave retrieval in layered media using seismic interferometry by multidimensional deconvolution: Geophysical Journal International, 196, 230-242, doi: 10 .1093/gji/ggt389.

Xia, J., R. D. Miller, and C. B. Park, 1999, Estimation of near-surface shear-wave velocity by inversion of Rayleigh waves: Geophysics, 64, 691-700, doi: 10.1190/ 1.1444578 .

Xia, J., R. D. Miller, C. B. Park, and Ivanov, 2004, Utilization of high-frequency Rayleigh waves in near-surface geophysics: The Leading Edge, 23, 753-759, doi: 10 $.1190 / 1.1786895$.

Xia, J., Y. Xu, and R. D. Miller, 2007, Generating an image of dispersive energy by frequency decomposition and slant stacking: Pure and Applied Geophysics, 164, 941-956, doi: 10.1007/s00024-007-0204-9.

Yilmaz, O., 1987, Seismic signal processing: SEG. 
Zhang, Z., Y. Liu, and G. Schuster, 2015, Wave equation inversion of skeletonized surface waves: 85th Annual International Meeting, SEG, Expanded Abstracts, 2391-2395.

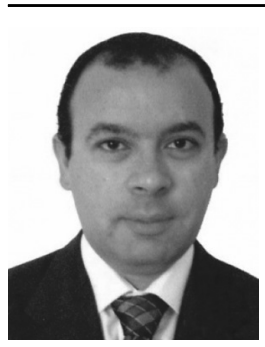

Sherif M. Hanafy received B.S. (1993) and master's (1996) degrees from Cairo University, Egypt, in geophysics and a Ph.D. (2002) from University of Kiel, Germany - Cairo University, Egypt. He has been a senior research scientist at KAUST since 2009 . He is in charge of the geophysical field program (Seismology Lab) and teaches geophysical field methods in addition to his research in interferometry, traveltime tomography, early arrival tomography, data interpolation/extrapolation, and shallow application of resistivity and GPR methods. He worked as associate professor at Cairo University, Egypt, from 2002 to 2007. In 2004, he received a one-year postdoc scholarship from Fulbright at University of Utah; in 2007-2009, he went back to Utah for the second time as a postdoc; and in 2009, he moved to KAUST as a senior research scientist. His main research interests include shallow application of geophysics for geologic, engineering, environmental, and archaeological application. He uses several geophysical methods in his work including seismic, electric, and GPR methods.

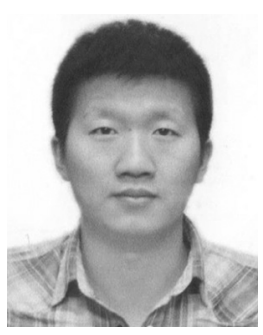

Jing Li received master's (2011) and Ph.D. (2014) degrees from Jilin University, China, in applied geophysics. He is a postdoc fellow at CSIM group, KAUST, where he focuses on seismic surface wave inversion and data processing. He has worked as a visiting researcher at Delaware State University, USA, from 2012 to 2014 . His work focused on the advance math method in geophysics application. His current research interests include seismic interferometry for subsurface monitoring and GPR simulation and inversion for a shallow subsurface, through-wall detection, and lunar radar detection. 